\title{
A Semiconductor Bridge Electrical Explosive Device Online Firing Quality Identification Algorithm
}

\author{
Bin-bin Zhang, Song Gao $\mathbb{D}^{D}$, Chao-bo Chen, Ji-chao Li, and Xue-qin Bi \\ School of Electronic Information Engineering, Xi'an Technological University, Xi'an 710021, Shaanxi, China \\ Correspondence should be addressed to Song Gao; gaos@xatu.edu.cn
}

Received 11 June 2020; Revised 10 September 2020; Accepted 29 October 2020; Published 21 November 2020

Academic Editor: Frederic Kratz

Copyright (C) 2020 Bin-bin Zhang et al. This is an open access article distributed under the Creative Commons Attribution License, which permits unrestricted use, distribution, and reproduction in any medium, provided the original work is properly cited.

\begin{abstract}
When qualified explosive devices fire the explosive agent unsuccessfully, on-site testers cannot diagnose fast and accurately whether it is the firing quality problem of the electrical explosive devices or explosive agent by using traditional test methods. And, if the explosive agent is fired unsuccessfully, generally, the only way is to test the explosive device by on-site testers themselves. In order to protect the on-site testers' safety, this paper proposes an electrical explosive device firing quality identification algorithm based on HHT (Hilbert-Huang transform) of the explosive time series. Obtaining an explosive current time series during the firing process of electrical explosive devices by the explosive equipment, the IMFs (intrinsic mode functions) and a residual function of the explosive current time series are obtained by EMD (empirical mode decomposition), the feature vector, which is the energy characteristic values of the IMFs and residual function by Hilbert transformation, is the input of SVM (support vector machine), and the fired failure explosive device is identified as an excellent performance product or performance failure product by the trained SVM. Finally, semiconductor explosive devices are tested to verify the proposed algorithm, and the results show that the EMD-SVM algorithm can identify effectively the firing quality of firing explosive devices.
\end{abstract}

\section{Introduction}

The mechanism of electrical explosive devices is to fire the resistance, semiconductor bridge, MEMS (micro-electromechanical system), and other hot-wire igniters by fire current excitation to make explosive agents work [1]. Comparing with other explosive devices, semiconductor bridge electrical explosive devices have the advantage of consistent synchronization, easy, and remote control [2]. So, it is most widely used in weapon, aerospace, and missile [3] applications.

Since the electrical explosive device is the initial ignition component of the weapon, the quality requirements of the electrical explosive device are very high. At present, the commonly used reliability test methods of explosive devices are mainly success or failure experiments: lifting method [4], Bootstrap method [5], 100\% $p$ firing level method [6], information measure equivalency [7], stress-strength interference method [8], and maximum likelihood estimation method [9]. But, these methods cannot identify precisely the firing quality of a single electrical explosive device [10]. Besides, some methods are based on the mathematic model, temperature rising model $[10,11]$, critical firing current model [12], finite element model [13], etc. However, the firing mathematic model of the explosive device is stochastic, and it does not completely fit the next explosive device. Moreover, the explosive devices are a product of "long-term storage and single use," and the explosive device test methods of literatures [4-13] are to test unused products in the storage environment. The explosive devices may fail, even if the batch of explosive devices is qualified, and may cause serious consequences such as misfire or an accidental explosion of weapons and equipment because of temperature, humidity, vibration, pressure, altitude, etc. [14]. Onsite testers are dangerous, when they test the failed explosive device, and cannot immediately determine whether there is a problem with the explosive device or explosive agent. So, the online firing quality identification algorithm of electrical explosive devices is actually needed at the test site, and it provides a basis for the intelligent firing or fault location of a 
weapon. But, online firing quality identification algorithms of explosive devices are very few.

With the development of digital technology, the electrical explosive equipment, which is based on an intelligent microprocessor, can obtain the time series of firing current and voltage, and it can be used to identify whether the fired failure explosive device can be used again. This paper mainly researches the explosive time series of semiconductor bridge electrical explosive devices. And, according to the impedance-energy curve, the explosive process is divided into positive impedance temperature rising, negative impedance temperature rising, melting, gasification, and plasma generation stages [15]. The dynamic impedance characteristic of the semiconductor bridge electrical explosive device is strongly nonlinear, time-varying, and strong stochastic because of the inconsistency of semiconductor material, temperature, humidity, installation error, explosive agent, etc. Its explosive mechanism is very complex, and the traditional impedance test or Fourier analysis method is not suitable for nonstationary time series.

In summary, the explosive current and voltage time series are obtained by the intelligent electrical explosive equipment. And, aiming at the strong nonlinear and nonstationary explosive time series, this paper gets the explosive characteristic value of time series by HHT, which is mainly composed of EMD and Hilbert transformation, and identifies the explosive quality by the trained SVM. As a result, the explosive failure explosive device can be determined online whether it can be used again, without the on-site testers identifying by themselves.

The organization of this paper is as follows. In Section 2, an online firing quality algorithm is proposed based on the EMD, Hilbert transformation, and SVM. And, in Section 3, 200 explosive current time series data sets are obtained by the electrical explosive equipment, and the effectiveness and feasibility of the firing quality identification algorithm is verified by those data sets. At last, some conclusions are drawn in Section 4.

\section{The Firing Quality Identification Methods of Explosive Device}

When the semiconductor bridge explosive device is ignited, it quickly heats up, the dynamic impedance curve of the firing explosive device is nonlinear and stochastic, and the explosive time series is nonstationary. The traditional methods, such as the impedance method, Fourier transformation method, and wavelet transformation method, are not fitted properly with the nonstationary time series. However, HHT (Hilbert-Huang transform) is a nonstationary analysis method widely used in recent years, and HHT is mainly composed of EMD and Hilbert transform [16]. The complex explosive time series can be decomposed into IMF components and a residual component by EMD, and they are independent of the sampling frequency; every IMF characteristic value is only related to the time series itself. So, EMD is an adaptive signal analysis method. And, the local Hilbert energy characteristic value can be obtained by the Hilbert transform of IMF components and the residual component to reduce the dimension of classification algorithms.

SVM is trained based on the Hilbert energy characteristic value of IMFs and the residual component, and the trained SVM can be used on the intelligent explosive equipment to identify the firing quality of explosive devices online.

2.1. EMD. The original time series can be decomposed into IMF components by EMD, and every IMF has to satisfy two conditions [16]:

(1) The number of extreme points is equal to the number of zero points, or their difference is 1

(2) The average value of the envelopes, which is formed by the maximum and minimum, is 0

The basic EMD method is used in this paper, and for detailed steps, refer the literature [16]. The EMD result of the original time series $x(t)$ is

$$
x(t)=\sum_{i=1}^{n} c_{i}(t)+r_{n}(t),
$$

where $x(t)$ is the original time series, $c_{i}(t)$ is the $i$ th IMF component, and $r_{n}(t)$ is the residual component. When $r_{n}(t)$ or $c_{n}(t)$ is less than a constant value or $r_{n}(t)$ is a monotonic function, the EMD stops.

2.2. Hilbert Transformation. Mutually orthogonal analytical signals can be obtained by the Hilbert transform of IMF components [17], and then the instantaneous amplitude and phase of each IMF are obtained. Hilbert transformation of IMF components is shown in the following equation:

$$
H(c(t))=\frac{1}{\pi t} * c(t)=\frac{1}{\pi} \int_{-\infty}^{+\infty} \frac{c(\tau)}{t-\tau} \mathrm{d} \tau,
$$

where ${ }^{*}$ is the convolution, $t$ is the time, and $\tau$ is the integral variable.

Analytical form of the IMF component is shown in the following equation:

$$
z(t)=c(t)+j H[c(t)]=a(t) e^{j \Phi(t)},
$$

where $a(t)$ is an amplitude function and $\Phi(t)$ is a phase function.

Then, the $i$ th energy characteristic value of the IMF component is

$$
E_{i}=\frac{\sum_{j=1}^{N}\left|a_{j}(t)\right|^{2}}{N}
$$

where $N$ is the number of IMF data points. At the same time, the energy characteristic value of the residual function is obtained by EMD and Hilbert transformation.

2.3. SVM. SVM is a commonly used classification algorithm in machine learning. The classification principle is shown in Figure 1; there are two samples in the figure, and they are represented by squares and circles, respectively. In the figure, 


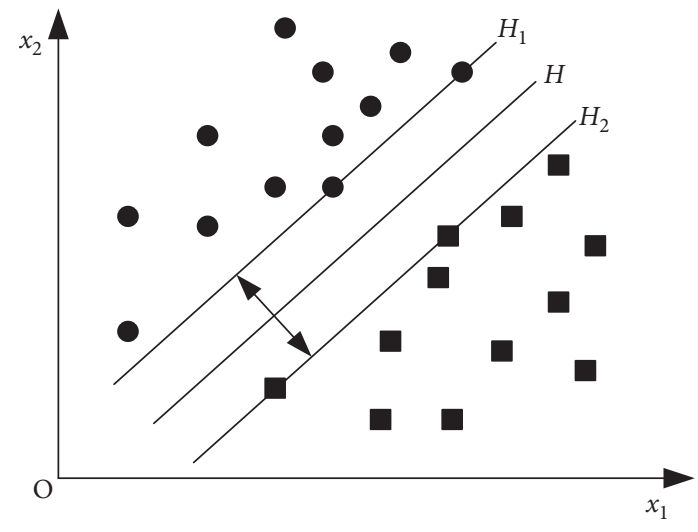

FIgUre 1: The schematic of SVM.

$H$ is the optimum classification line of two samples, $H_{1}$ and $\mathrm{H}_{2}$, which are parallel to the classification line $H$ and pass through the sample closest to the classification line [18]. The goal of SVM is to construct the classification function $H$ so that the classification interval of $H_{1}$ and $H_{2}$ is the largest.

SVM of this paper is a two-class classification problem, and suppose that the training set of SVM is $T=\left\{x_{k}, y_{k}\right\}$, where $k=1,2,3, \ldots, m, x_{k} \in \mathbf{R}^{n}$, and $y_{k}=\{-1,1\}$. And, the classification function is

$$
g(x)=w x+b,
$$

where $w$ is the weight vector and $b$ is the threshold.

Considering that there may be a linear inseparable situation in reality, the relaxation variable $\xi_{i}$ and the penalty factor $C$ are introduced, so the SVM problem transforms a convex optimization problem, as shown in (6). The convex optimization problem can be transformed to solve the dual quadratic programming problem, and the detailed solving steps can be referenced from [18]:

$$
\begin{cases}\min _{w, b} & \frac{1}{2}\|w\|^{2}+C \sum_{i=1}^{m} \xi_{i}, \\ \text { s.t. } \quad & y_{i}\left(w_{i}^{T} x_{i}+b\right)>1-\xi_{i}(i=1,2, \ldots, m) .\end{cases}
$$

2.4. The Firing Quality Identification Algorithm of Explosive Device Based on EMD-SVM. In reality, explosive devices may be degradation or failure, when the explosive agent does not work. And, the explosive device of degradation can be used again by increasing the firing voltage, and the explosive device of failure cannot fire the explosive agent forever. So, the explosive device of degradation is still excellent performance, and the failure explosive device is still performance failure. The goal of the explosive device firing quality identification algorithm is to find whether there is a problem with the explosive device or other, and it is a two-class classification problem.

Generally, semiconductor bridge explosive devices are fired by DC power supply or capacitor energy storage, and this paper only chooses the explosive current time series as the identification signal because the explosive voltage time series keeps almost constant or decreases and does not meet
EMD requirement [19]. The firing quality identification algorithm of the semiconductor explosive device is shown in Figure 2. At first, the explosive current time series is obtained on site, normalized, and the IMF components and residual component are obtained by EMD; the explosive characteristic values can be obtained by Hilbert transformation to train SVM; and last of all, the trained SVM can be used to identify whether there is a problem with the explosive device or other. The detailed steps are as follows:

Step 1: get the original explosive current time series, including the performance failure and excellent performance signal. Then, the performance of the fired explosive device can be obtained by ex post analysis of the experimenter. Moreover, the explosive current time series is normalized to get $X(t)$.

Step 2: EMD of $X(t)$ and obtaining the IMF components and residual component.

Step 3: the ith energy characteristic value $E i$ of IMF and the residual energy characteristic value ER of the residual component are obtained by Hilbert transformation, and $E i$ and ER are added to get $E$. Secondly, the energy characteristic vector is constructed as in (7), where $M$ is the number of IMF components:

$$
\left(\frac{E_{1}}{E}, \frac{E_{2}}{E}, \ldots, \frac{E_{M}}{E}, \frac{E_{R}}{E}\right) .
$$

Step 4: the explosive time series result $\mathrm{yk}=1$, if the performance of the semiconductor explosive device is ok. Otherwise, $y k=-1$. At last, the energy characteristic vector and yk are the training set of SVM, and SVM training is completed.

Step 5: verifying the trained SVM by the test set.

\section{Experiment Analyses}

3.1. Explosive Firing Equipment. This paper takes the type of semiconductor bridge explosive devices with relatively complicated dynamic impedance as an example; these explosive devices are explosive bolts, their firing sustaining time is $100 \mathrm{~ms}$, and their firing voltage is $12 \mathrm{~V}$. Because the explosive devices have not been fully finalized, the failure probability of explosive devices is still high, when they detonate the explosive agent. The structure of the explosive equipment is shown in Figure 3, and it detonates semiconductor explosive devices by supercapacitor charging and discharging. The diodes of this explosive equipment are used to prevent each detonating branch affecting each other, the charging switches are used to charge the supercapacitors, the current limiting resistors are used to limit the charging current of super capacitors too large, and the ignition switches are used to fire the explosive devices. At the same time, the explosive equipment can obtain the explosive current and voltage of explosive devices. The internal diagram of the explosive equipment is shown in Figure 4, and the $\mathrm{AD}$ sample frequency is $10 \mathrm{MHz}$, and it can meanwhile fire 8 explosive devices. 


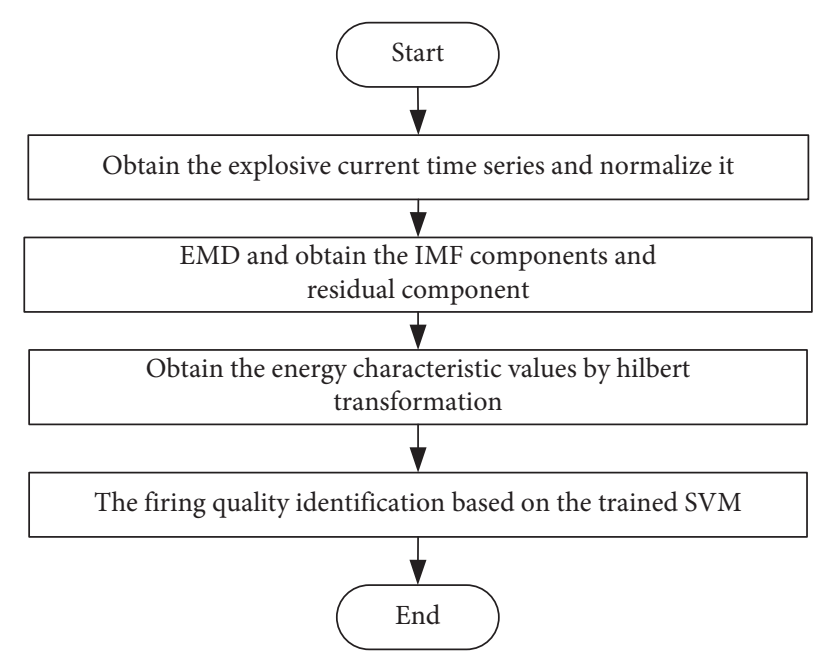

Figure 2: The firing qualification identification flowchart of electrical explosive devices.

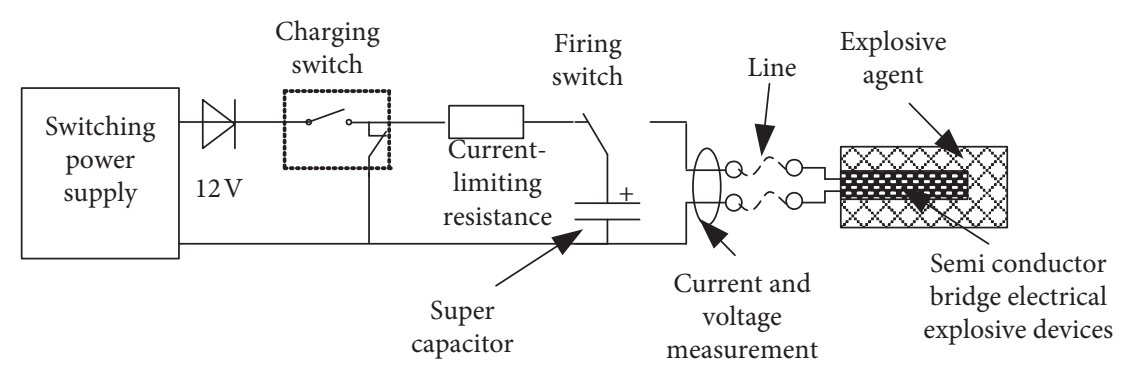

FIGURE 3: The schematic of the explosive firing equipment.

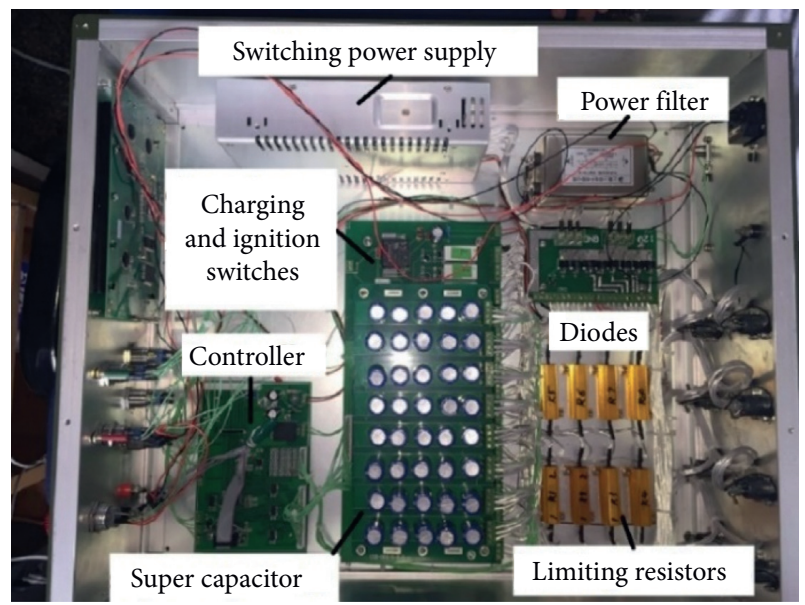

FIGURE 4: The semiconductor bridge explosive firing equipment.

3.2. Explosive Time Series. In the novelty explosive bolt test, if the explosive agent fires unsuccessfully, the explosive device may be used again. So, there are excellent performance and performance failure explosive devices. And, two explosive current and voltage time series of excellent performance and performance failure explosive devices are shown in Figure 5, and each explosive time series has 5000 sampling points. From Figure 5, the explosive voltage curves are basically unchanged; however, the explosive current curves show obvious difference, so the explosive current is chosen.

From the explosive current curves, it can be seen that they are nonlinear and nonstationary signals. So, the traditional impedance method or Fourier method is not suitable for nonstationary signals, and the HHT method, which is composed of EMD and Hilbert transform, can effectively analyze the explosive current time series to reduce the nonlinear and nonstationary characteristics of explosive time series $[20,21]$.

3.3. EMD and Hilbert Transformation. Explosive current time series of excellent performance and performance failure explosive devices is normalized; moreover, the EMD results of the normalized explosive current time series are shown in Figure 6. The residual component amplitude of excellent performance explosive devices is larger than performance failure explosive devices. And, the amplitude of IMF components is obviously different between excellent performance and performance failure explosive devices. So, the IMF components and residual component can be used to identify whether the explosive device shows excellent performance or performance failure. Moreover, the Hilbert energy characteristic of IMFs and residual components can be obtained to reduce the input dimensions of classification algorithms [22, 23]. 


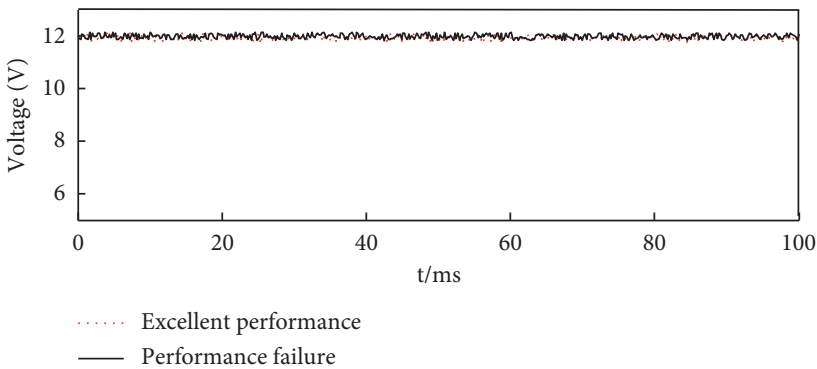

(a)

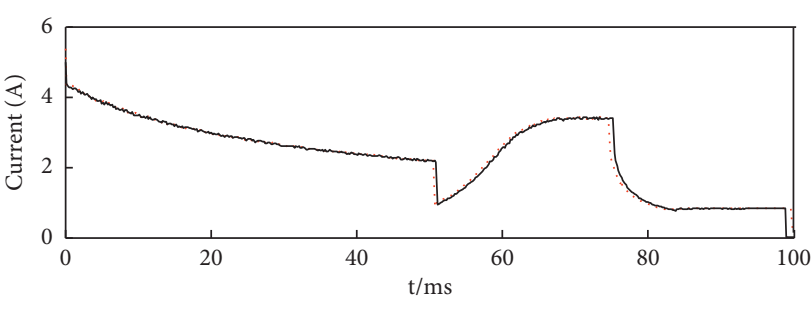

Excellent performance — Performance failure

FIgURE 5: The voltage (a) and current (b) curves of normal and abnormal firing semiconductor bridge explosive devices.
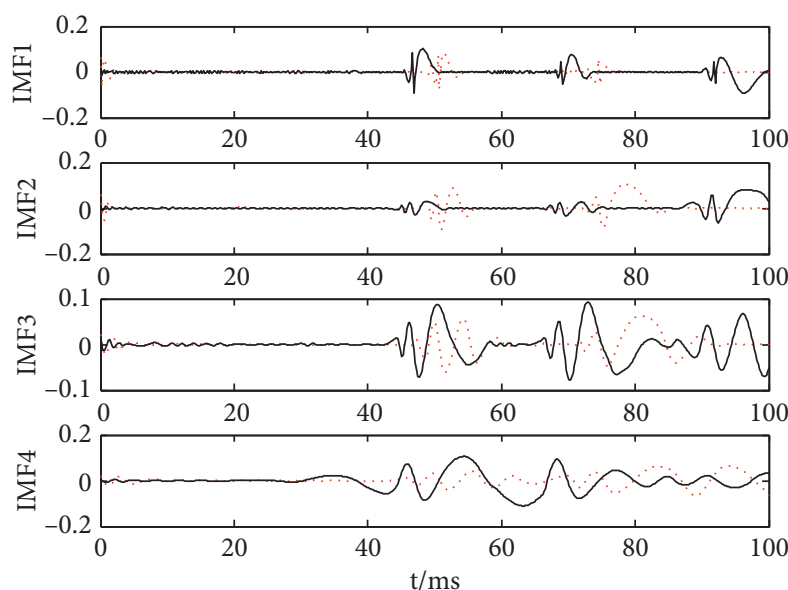

Excellent performance

_ Performance failure

(a)
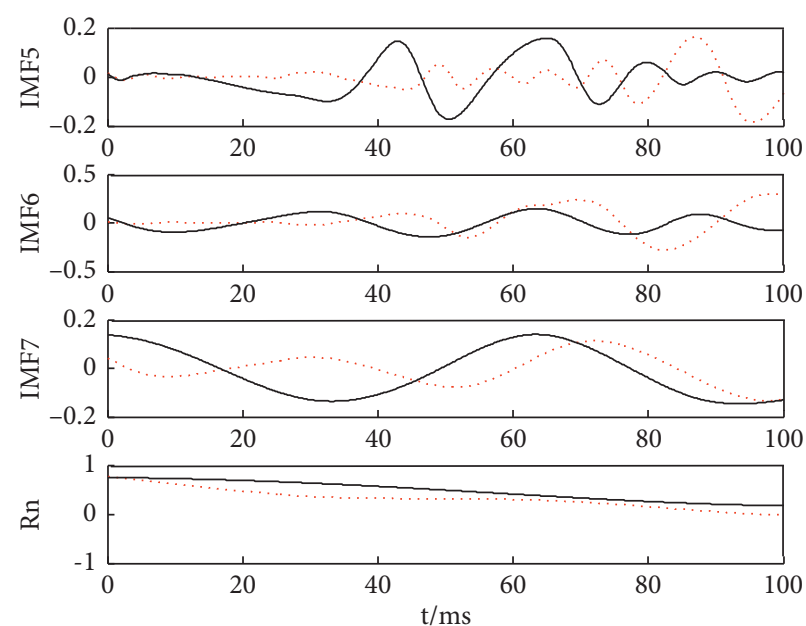

Excellent performance

- Performance failure

(b)

FiguRE 6: The EMD results of abnormal and normal semiconductor bridge explosive devices firing. (a) The result of IMF1 IMF4. (b) The result of IMF5 IMF7 and the residual component.

The energy characteristic values of IMF components and the residual component are obtained by Hilbert transformation as shown in Table 1, and the energy characteristic values are different between IMF components and the residual component of excellent performance and performance failure devices. E2 E7 and $\mathrm{E}_{\mathrm{R}}$ are obviously different, and they can be used as input variables of classification algorithms.

3.4. Classification. In this paper, the energy characteristics of the IMFs and residual function are inputs of classification algorithms, and the SVM method is chosen as the classification algorithm to compare with KNN (K-near neighbor) and naive Bayes [19]. And, the relaxation variable $\xi \mathrm{i}$ and the penalty factor $C$ of SVM can be optimized by PSO (particle swarm optimization) [23] or other intelligent optimization algorithms $[24,25]$.

The manufacturer provides 200 explosive devices' test data. There are 100 explosive devices with excellent performance and others are performance failure. This paper
TABLE 1: The energy characteristics of the IMF and residual function.

\begin{tabular}{lcc}
\hline Energy & Excellent performance & Performance failure \\
\hline E1 & 0.0003 & 0.0004 \\
E2 & 0.0013 & 0.0007 \\
E3 & 0.0007 & 0.0016 \\
E4 & 0.0012 & 0.0033 \\
E5 & 0.0076 & 0.0112 \\
E6 & 0.0356 & 0.0136 \\
E7 & 0.0078 & 0.0201 \\
E $_{R}$ & 0.1768 & 0.3011 \\
\hline
\end{tabular}

selects 50 sets of excellent performance signals and 50 sets of performance failure signals as the training set and others are the test set. The energy characteristics of the IMFs and residual function are inputs of the classification algorithm. And, the results of 3 classification algorithms are shown in Table 2. The accuracy rating of the proposed algorithm is larger than $\mathrm{KNN}$ and naive Bayes methods. F1-score is commonly used in classification problems or an information 
TABLE 2: The identification results of 3 classification algorithms.

\begin{tabular}{|c|c|c|c|c|c|}
\hline Classification algorithm & Data sample & Correct times & Failure times & Accuracy rating (\%) & F1-score \\
\hline \multirow{2}{*}{ SVM } & Excellent performance & 47 & 3 & 94 & \multirow{2}{*}{0.9307} \\
\hline & Performance failure & 46 & 4 & 92 & \\
\hline \multirow{2}{*}{$\mathrm{KNN}$} & Excellent performance & 47 & 3 & 94 & \multirow{2}{*}{0.9216} \\
\hline & Performance failure & 45 & 5 & 90 & \\
\hline \multirow{2}{*}{ Naive Bayes } & Excellent performance & 46 & 4 & 92 & \multirow{2}{*}{0.9109} \\
\hline & Performance failure & 45 & 5 & 90 & \\
\hline
\end{tabular}

retrieval system, and the detailed method of F1-score is introduced in [26]. If the F1-score is higher, the classification effect is better [26]. F1-score of the proposed method is 0.9307, and it shows that the proposed methods can be effectively used in explosive device quality classification.

\section{Conclusion}

This paper proposes a firing quality identification algorithm to quickly identify whether there is a problem with explosive devices or other, to avoid on-site testers to personally check the failed firing explosive devices, and in order to keep them safe.

This semiconductor bridge electrical explosive device online firing quality identification algorithm decomposes the explosive current time series by EMD, obtains the energy characteristic values by Hilbert transformation of the IMF components and residual component, and in the end, the trained SVM is used to identify the firing quality of explosive devices. The identification results show the effectiveness and feasibility of the algorithm, and the firing identification algorithm can provide a basis for the evaluation of the firing quality of most electrical explosive devices, provide support for fault location of weapons and explosive systems, improvement of the storage environment of explosive devices, etc., and protect the safety of testers.

However, the SVM is linear, the identification results may not be accurate, and a deep learning algorithm is the future research direction.

\section{Data Availability}

The explosive voltage and current time series data used to support the findings of this study are available from the corresponding author upon request.

\section{Conflicts of Interest}

The authors declare that they have no conflicts of interest.

\section{Acknowledgments}

This work was supported by the Shaanxi Provincial Department of Education Natural Science Special Scientific Research Plan Project (17JK1069).

\section{References}

[1] Y. Bai, W. Ren, J. Liu et al., "Relationship between continuous electromagnetic wave frequency and EED induced current,"
Chinese Journal of Energetic Materials, vol. 22, no. 4, pp. 521-524, 2014.

[2] W. J. Dixon and A. M. Mood, "A method for obtaining and analyzing sensitivity data," Journal of the American Statistical Association, vol. 43, no. 241, pp. 109-126, 1948.

[3] X. Xu, J. Li, X. Wei et al., "Study on the aging effect of a detonator," Chinese Journal of Energetic Materials, vol. 16, no. 5, pp. 539-542, 2008.

[4] H. Dong, X. Dong, T. F. Zhang et al., "Reliability verification of initiating devices based on rigorous test," Transactions of Beijing Institute of Technology, vol. 33, no. 3, pp. 221-224, 2013.

[5] Y. Wen, L. Zhang, and D. Hong, "Analysis on reliability for explosive initiator based on sensitivity," Acta Armamentarii, vol. 31, no. 11, pp. 1498-1501, 2010.

[6] Y. Tian and D. Wang, "A qualification test method for firing reliability of initiator with small samples," Acta Armamentarii, vol. 32, no. 4, pp. 426-431, 2011.

[7] H. Dong, X. Zhao, and R. Cai, "An assessment method of reliability of initiating devices based on information measure equivalency," Acta Armamentarii, vol. 32, no. 05, pp. 554-558, 2011.

[8] X. Yi, H. Dong, Z. Zhai et al., "Reliability design for initiating devices based on stress-strength interference model," Transactions of Beijing Institute of Technology, vol. 34, no. 10, pp. 1007-1011, 2014.

[9] S. Guoqing, G. Xiaoguang, L. Mingyang et al., "Research on the safety response rule of initiating explosive device," in Proceedings of 2011 IEEE 2nd International Conference on Computing, Control and Industrial Engineering, vol. 2, pp. 147-150, IEEE, Wuhan, China, 2011.

[10] M. Liu and X. Zhang, "Temperature rising characteristic of bridge in semiconductor bridge," Journal of Nanjing University of Science and Technology (Natural Science), vol. 34, no. 02, pp. 203-206+212, 2010.

[11] J. Cao, R. Cai, H. Dong et al., "The temperature rising models of bridgewire and explosive of electro-explosive device and application in reliability design," Explosion and Shock Waves, vol. 24, no. 01, pp. 90-95, 2004.

[12] Q. Zhou and Q. Jiao, "Firing model of Ni-Cr bridge-belt electric initiating devices under current input," Chinese Journal of Energetic Materials, vol. 22, no. 06, pp. 824-827, 2014.

[13] H. Li, Y. Bai, W. Ren et al., "Simulation research on temperature besponse characteristics on initiating device transformer under DC excitation," Acta Armamentarii, vol. 37, no. S2, pp. 138-143, 2016.

[14] R. Zhang, L. I. Fang, Z. Dou et al., "Research on storage characteristics of electric explosive devices under single temperature storage condition," Acta Armamentarii, vol. 37, no. S2, pp. 138-143, 2016. 
[15] M. Zhu, B. He, M. Hu et al., "Study on dynamic resistance of semiconductor bridge," Explosive Materials, vol. 36, no. 02, pp. 18-20, 2007.

[16] N. E. Huang, Z. Shen, S. R. Long et al., "The empirical mode decomposition and the Hilbert spectrum for nonlinear and non-stationary time series analysis," Proceedings of the Royal Society of London. Series A: Mathematical, Physical and Engineering Sciences, vol. 454, no. 1971, pp. 903-995, 1998.

[17] E. Elbouchikhi, V. Choqueuse, Y. Amirat, M. E. H. Benbouzid, and S. Turri, "An efficient hilbert-huang transform-based bearing faults detection in induction machines," IEEE Transactions on Energy Conversion, vol. 32, no. 2, pp. 401-413, 2017.

[18] S. Boyd and L. Vandenberghe, Convex optimization, Cambridge University Press, Cambridge, UK, 2004.

[19] R. Sharma and R. B. Pachori, "Classification of epileptic seizures in EEG signals based on phase space representation of intrinsic mode functions," Expert Systems with Applications, vol. 42, no. 3, pp. 1106-1117, 2015.

[20] Z. Zhang, W.-C. Hong, and J. Li, "Electric load forecasting by hybrid self-recurrent support vector regression model with variational mode decomposition and improved cuckoo search algorithm," IEEE Access, vol. 8, pp. 14642-14658, 2020.

[21] G.-F. Fan, L.-L. Peng, W.-C. Hong, and F. Sun, "Electric load forecasting by the SVR model with differential empirical mode decomposition and auto regression," Neurocomputing, vol. 173, pp. 958-970, 2016.

[22] Z. Zhang and W.-C. Hong, "Electric load forecasting by complete ensemble empirical mode decomposition adaptive noise and support vector regression with quantum-based dragonfly algorithm," Nonlinear Dynamics, vol. 98, no. 2, pp. 1107-1136, 2019.

[23] M.-W. Li, J. Geng, W.-C. Hong, and L.-D. Zhang, "Periodogram estimation based on LSSVR-CCPSO compensation for forecasting ship motion," Nonlinear Dynamics, vol. 97, no. 5, pp. 2579-2594, 2019.

[24] W.-C. Hong, "Electric load forecasting by seasonal recurrent SVR (support vector regression) with chaotic artificial bee colony algorithm," Fuel and Energy Abstracts, vol. 36, no. 9, pp. 5568-5578, 2011.

[25] W.-C. Hong, Y. Dong, W. Y. Zhang, L.-Y. Chen, and B. K. Panigrahi, "Cyclic electric load forecasting by seasonal SVR with chaotic genetic algorithm," International Journal of Electrical Power \& Energy Systems, vol. 44, no. 1, pp. 604-614, 2013.

[26] H. Huang, H. Xu, X. Wang, and W. Silamu, "Maximum F1score discriminative training criterion for automatic mispronunciation detection," IEEE/ACM Transactions on Audio, Speech, and Language Processing, vol. 23, no. 4, pp. 787-797, 2015. 